\title{
Mycobacterium tuberculosis H37Rv: In Silico Drug Targets Identification by Metabolic Pathways Analysis
}

\author{
Asad Amir, Khyati Rana, Arvind Arya, Neelesh Kapoor, \\ Hirdesh Kumar, and Mohd Asif Siddiqui \\ Department of Biotechnology, Meerut Institute of Engineering and Technology, N.H. 58, Delhi-Roorkee Highway, \\ Baghpat Road Bypass Crossing, Meerut 250005, India
}

Correspondence should be addressed to Asad Amir; asadbioinfo@gmail.com

Received 10 May 2013; Revised 26 October 2013; Accepted 26 December 2013; Published 25 February 2014

Academic Editor: Graziano Pesole

Copyright (C) 2014 Asad Amir et al. This is an open access article distributed under the Creative Commons Attribution License, which permits unrestricted use, distribution, and reproduction in any medium, provided the original work is properly cited.

\begin{abstract}
Mycobacterium tuberculosis (Mtb) is a pathogenic bacteria species in the genus Mycobacterium and the causative agent of most cases of tuberculosis. Tuberculosis (TB) is the leading cause of death in the world from a bacterial infectious disease. This antibiotic resistance strain lead to development of the new antibiotics or drug molecules which can kill or suppress the growth of Mycobacterium tuberculosis. We have performed an in silico comparative analysis of metabolic pathways of the host Homo sapiens and the pathogen Mycobacterium tuberculosis (H37Rv). Novel efforts in developing drugs that target the intracellular metabolism of $M$. tuberculosis often focus on metabolic pathways that are specific to $M$. tuberculosis. We have identified five unique pathways for Mycobacterium tuberculosis having a number of 60 enzymes, which are nonhomologous to Homo sapiens protein sequences, and among them there were 55 enzymes, which are nonhomologous to Homo sapiens protein sequences. These enzymes were also found to be essential for survival of the Mycobacterium tuberculosis according to the DEG database. Further, the functional analysis using Uniprot showed involvement of all the unique enzymes in the different cellular components.
\end{abstract}

\section{Introduction}

Mycobacterium tuberculosis (Mtb), the causative agent of tuberculosis (TB), remains a major health threat. Each year, 8 million new TB cases appear and 2 million individuals die of TB [1]. Further, about half a million new multidrug resistant TB cases are estimated to occur every year [2]. The existing drugs, although of immense value in controlling the disease to the extent that is being done today, have several shortcomings, the most important of them being the emergence of drug resistance rendering even the frontline drugs inactive. In addition, drugs such as rifampicin have high levels of adverse effects making them prone to patient incompliance. Another important problem with most of the existing antimycobacterials is their inability to act upon latent forms of the bacillus. In addition to these problems, the vicious interactions between the HIV (human immunodeficiency virus) and TB have led to further challenges for antitubercular drug discovery [3].

Recently, genome-scale metabolic network reconstructions for different organisms have enabled systematic analyses of metabolic functions and predictions of metabolismrelated phenotypes. By collecting all possible biochemical reactions for specific organisms, different groups have reconstructed metabolic networks for bacteria, for example, Escherichia coli, Helicobacter pylori, and Chromohalobacter salexigens, eukaryotic microorganisms, mice, and even humans [4-6]. The website of the Systems Biology Research Group at the University of California, San Diego (http://gcrg.ucsd.edu/), provides a continuously updated list of genome-scale metabolic network reconstructions. Analysis of metabolic networks can provide insights into an organism's ability to grow under specific conditions. For example, given a specific set of nutrient conditions, flux 
TABLE 1: Unique pathways of $M$. tuberculosis when compared to H. sapiens.

\begin{tabular}{|c|c|c|c|}
\hline S. no. & Pathway name & Human & Mycobacterium tuberculosis $\mathrm{H} 37 \mathrm{Rv}$ \\
\hline 1 & Carbohydrate Metabolism & & \\
\hline 1.1 & C5-Branched dibasic acid metabolism & Absent & Present \\
\hline 2 & Energy Metabolism & & \\
\hline 2.1 & Photosynthesis & Absent & Absent \\
\hline 2.2 & Carbon fixation pathways in prokaryotes & Absent & Present \\
\hline 2.3 & Methane metabolism & Absent & Present \\
\hline 3 & Lipid Metabolism & & \\
\hline 3.1 & Fatty acid elongation in mitochondria & Present & Absent \\
\hline 3.2 & Sphingolipid metabolism & Present & Absent \\
\hline 3.3 & Arachidonic acid metabolism & Present & Absent \\
\hline 4 & Nucleotide Metabolism & All Present & All Present \\
\hline 5 & Amino Acid Metabolism & All Present & All Present \\
\hline 6 & Metabolism of Other Amino Acids & All Present & All Present \\
\hline 6.1 & Phosphonate and phosphinate metabolism & Absent & Absent \\
\hline 7 & Glycan Biosynthesis and Metabolism & & \\
\hline 7.1 & N-Glycan biosynthesis & Present & Absent \\
\hline 7.2 & Various types of $\mathrm{N}$-glycan biosynthesis & & Absent \\
\hline 7.3 & Mucin type O-Glycan biosynthesis & Present & Absent \\
\hline 7.4 & Other types of O-glycan biosynthesis & Present & Absent \\
\hline 7.5 & Glycosaminoglycan biosynthesis_-chondroitin sulfate & Present & Absent \\
\hline 7.6 & Glycosaminoglycan biosynthesis-heparan sulfate & Present & Absent \\
\hline 7.7 & Glycosaminoglycan biosynthesis-keratan sulfate & Present & Absent \\
\hline 7.8 & Glycosaminoglycan degradation & Present & Absent \\
\hline 7.9 & Glycosylphosphatidylinositol (GPI)-anchor biosynthesis & Present & Absent \\
\hline 7.10 & Glycosphingolipid biosynthesis_lacto and neolacto series & Present & Absent \\
\hline 7.11 & Glycosphingolipid biosynthesis-globo series & Present & Absent \\
\hline 7.12 & Glycosphingolipid biosynthesis-ganglio series & Present & Absent \\
\hline 7.13 & Lipopolysaccharide biosynthesis & Absent & Present \\
\hline 7.14 & Peptidoglycan biosynthesis & Absent & Present \\
\hline 7.15 & Other Glycan degradation & Present & Absent \\
\hline
\end{tabular}

balance analysis (FBA) of metabolic networks can accurately predict microbial cellular growth rates. In a recent work, a group of researchers used an approximate representation of in-host nutrient availability inferred from the literature to simulate the in-host metabolism of Salmonella typhimurium [7]. Moreover, metabolic network analyses can then be used to identify organism-specific essential genes by predicting the attenuation of microbial growth of specific deletion mutants [8-10].

The computational approach has been used to investigate novel drug targets in other pathogenic organisms such as Pseudomonas aeruginosa and in Helicobacter pylori $[5,11]$.

As most currently known, antibacterials are essentially inhibitors of certain bacterial enzymes; all enzymes specific to bacteria can be considered as potential drug targets [12]. In this study, we have adopted a strategy for comparative metabolic pathway analysis to find out some potential targets against $M$. tuberculosis (H37Rv). Only those enzymes which show unique properties than the host were selected as the target. Metabolic genes that are essential for pathogen growth but are not present in humans constitute actual and potential drug targets.

\section{Materials and Methods}

KEGG (Kyoto Encyclopedia of Gene and Genome) (http:// www.genome.jp/pathways.html) [13] pathway database was used as a source of metabolic pathway information. Metabolic pathway identification numbers of the host $H$. sapiens and the pathogen $M$. tuberculosis $(H 37 R v)$ were extracted from the KEGG database. Pathways which do not appear in the host but are present in the pathogen according to KEGG database have been identified as pathways unique to $M$. tuberculosis as in comparison to the host $H$. sapiens. Enzymes in these unique pathways as well as enzymes involved in other metabolic pathways under carbohydrate metabolism, energy metabolism, lipid metabolism, nucleotide metabolism, amino acid metabolism, metabolism of other amino acids, and glycan biosynthesis were identified from the KEGG database. The corresponding protein sequences of enzymes involved in unique pathways were 
TABle 2: Essential enzymes using DEG.

\begin{tabular}{|c|c|c|c|}
\hline S. no. & Entry no. & Protein name & Essential enzyme \\
\hline 1. & Rv1820 & Acetolactate synthase & Yes \\
\hline 2. & Rv0951 & Succinyl-CoA synthetase subunit beta & Yes \\
\hline 3. & Rv2987c & Isopropylmalate isomerase small subunit & Yes \\
\hline 4. & Rv1475c & Aconitate hydratase (EC: 4.2.1.3) & Yes \\
\hline 5. & Rv0066c & Isocitrate dehydrogenase (EC: 1.1.1.42) & Yes \\
\hline 6. & Rv2454c & 2-Oxoglutarate ferredoxin oxidoreductase subunit beta (EC: 1.2.7.3) & Yes \\
\hline 7. & Rv1240 & Malate dehydrogenase (EC: 1.1.1.37) & Yes \\
\hline 8. & Rv1098c & Fumarate hydratase (EC: 4.2.1.2) & Yes \\
\hline 9. & Rv0247c & Fumarate reductase iron-sulfur subunit (EC: 1.3.99.1) & Yes \\
\hline 10. & Rv3356c & $\begin{array}{l}\text { Bifunctional 5,10-methylene-tetrahydrofolate } \\
\text { dehydrogenase/5,10-methylene-tetrahydrofolate Cyclohydrolase (EC: } 1.5 .1 .5 \text { 3.5.4.9) }\end{array}$ & Yes \\
\hline 11. & Rv0951 & Succinyl-CoA synthetase subunit beta (EC: 6.2.1.5) & Yes \\
\hline 12. & Rv0904c & Putative acetyl-coenzyme A carboxylase carboxyl transferase subunit beta (EC: 6.4.1.2) & Yes \\
\hline 13. & Rv0973c & Acetyl-/propionyl-coenzyme A carboxylase subunit alpha (EC: 6.3.4.14) & Yes \\
\hline 14. & Rv1492 & Methylmalonyl-CoA mutase small subunit (EC: 5.4 .99 .2$)$ & Yes \\
\hline 15. & Rv3667 & Acetyl-CoA synthetase (EC: 6.2.1.1) & Yes \\
\hline 16. & Rv0409 & Acetate kinase (EC: 2.7.2.1) & Yes \\
\hline 17. & Rv0408 & Phosphate acetyltransferase (EC: 2.3.1.8) & Yes \\
\hline 18. & Rv0243 & Acetyl-CoA acetyltransferase (EC: 2.3.1.9) & Yes \\
\hline 19. & Rv0860 & Fatty oxidation protein FadB & Yes \\
\hline 20. & Rv3667 & Acetyl-CoA synthetase (EC: 6.2.1.1) & Yes \\
\hline 21. & Rv0373c & Carbon monoxyde dehydrogenase large subunit (EC: 1.2.99.2) & No \\
\hline 22. & Rv2900c & Formate dehydrogenase H (EC: 1.2.1.2) & No \\
\hline 23. & Rv1023 & Phosphopyruvate hydratase (EC: 4.2.1.11) & Yes \\
\hline 24. & Rv1240 & Malate dehydrogenase (EC: 1.1.1.37) & Yes \\
\hline 25. & Rv0070c & Serine hydroxymethyltransferase (EC: 2.1.2.1) & Yes \\
\hline 26. & Rv2205c & Hypothetical protein & Yes \\
\hline 27. & Rv0761c & Zinc-containing alcohol dehydrogenase NAD dependent AdhB (EC: 1.1.1.1) & Yes \\
\hline 28. & Rv0489 & Phosphoglyceromutase (EC: 5.4 .2 .1$)$ & Yes \\
\hline 29. & Rv0363c & Fructose-bisphosphate aldolase (EC: 4.1.2.13) & Yes \\
\hline 30. & Rv2029c & Phosphofructokinase PfkB (phosphohexokinase) (EC: 2.7.1.-) & Yes \\
\hline 31. & Rv1908c & Catalase-peroxidase-peroxynitritase T KatG (EC: 1.11.1.6) & Yes \\
\hline 32. & Rv0070c & Serine hydroxymethyltransferase (EC: 2.1 .2 .1 ) & Yes \\
\hline 33. & Rv0728c & D-3-phosphoglycerate dehydrogenase (EC: 1.1.1.95) & Yes \\
\hline 34. & Rv0505c & Phosphoserine phosphatase (EC: 3.1 .3 .3 ) & Yes \\
\hline 35. & Rv0884c & Phosphoserine aminotransferase (EC: 2.6.1.52) & Yes \\
\hline 36. & Rv0409 & Acetate kinase (EC: 2.7.2.1) & Yes \\
\hline 37. & Rv0408 & Phosphate acetyltransferase (EC: 2.3.1.8) & Yes \\
\hline 38. & Rv3667 & Acetyl-CoA synthetase (EC: 6.2.1.1) & Yes \\
\hline 39. & Rv2611c & Lipid A biosynthesis lauroyl acyltransferase (EC: 2.3.1. - ) & Yes \\
\hline 40. & Rv0114 & D-alpha,beta-D-heptose-1,7-biphosphate phosphatase (EC: 2. -...-) & Yes \\
\hline 41. & Rv0113 & Phosphoheptose isomerase (EC: 5. -...-) & Yes \\
\hline 42. & Rv1315 & UDP-N-acetylglucosamine 1-carboxyvinyltransferase (EC: 2.5.1.7) & Yes \\
\hline 43. & Rv0482 & UDP-N-acetylenolpyruvoylglucosamine reductase (EC: 1.1.1.158) & Yes \\
\hline 44. & Rv2152c & UDP-N-acetylmuramate-L-alanine ligase (EC: 6.3.2.8) & Yes \\
\hline 45. & Rv2155c & UDP-N-acetylmuramoyl-L-alanyl-D-glutamate synthetase (EC: 6.3.2.9) & Yes \\
\hline 46. & Rv2157c & $\begin{array}{l}\text { UDP-N-acetylmuramoylalanyl-D-glutamyl-2,6-diaminopimelate-D-alanyl-D-alanyl } \\
\text { ligase MurF }\end{array}$ & Yes \\
\hline
\end{tabular}


TABLE 2: Continued.

\begin{tabular}{|c|c|c|c|}
\hline S. no. & Entry no. & Protein name & Essential enzyme \\
\hline 47. & Rv2156c & Phospho-N-acetylmuramoyl-pentapeptide-transferase (EC: 2.7.8.13) & Yes \\
\hline 48. & Rv2153c & $\begin{array}{l}\text { Undecaprenyldiphospho-muramoylpentapeptide } \\
\text { beta-N-acetylglucosaminyltransferase (EC: } 2.4 .1 .227 \text { ) }\end{array}$ & Yes \\
\hline 49. & Rv2911 & D-alanyl-D-alanine carboxypeptidase (EC: 3.4 .16 .4 ) & No \\
\hline 50. & Rv2981c & D-alanyl-alanine synthetase A (EC: 6.3.2.4) & Yes \\
\hline 51. & Rv2136c & Undecaprenyl pyrophosphate phosphatase (EC: 3.6 .1 .27$)$ & Yes \\
\hline 52. & Rv2911 & D-alanyl-D-alanine carboxypeptidase (EC: 3.4 .16 .4 ) & No \\
\hline 53. & Rv2158c & UDP-N-acetylmuramoylalanyl-D-glutamate-2,6-diaminopimelate ligase (EC: 6.3.2.13) & Yes \\
\hline 54. & Rv2157c & $\begin{array}{l}\text { UDP-N-acetylmuramoylalanyl-D-glutamyl-2,6-diaminopimelate-D-alanyl-D-alanyl } \\
\text { ligase MurF }\end{array}$ & Yes \\
\hline 55. & Rv2156c & Phospho-N-acetylmuramoyl-pentapeptide-transferase (EC: 2.7.8.13) & Yes \\
\hline 56. & Rv2153c & $\begin{array}{l}\text { Undecaprenyldiphospho-muramoylpentapeptide } \\
\text { beta-N-acetylglucosaminyltransferase (EC: 2.4.1.227) }\end{array}$ & Yes \\
\hline 57. & Rv3910 & Transmembrane protein & Yes \\
\hline 58. & Rv0016c & Penicillin-binding protein $\mathrm{PbpA}$ & Yes \\
\hline 59. & Rv2163c & Penicillin-binding membrane protein $\mathrm{PbpB}$ & Yes \\
\hline 60. & Rv2911 & D-alanyl-D-alanine carboxypeptidase (EC: 3.4 .16 .4 ) & No \\
\hline
\end{tabular}

identified and their protein sequences were retrieved in FASTA format from KEGG database.

The unique enzymes were further analyzed for essentiality to pathogen by DEG (Database of Essential Genes) database (http://tubic.tju.edu.cn/deg/) [14], and considered cutoff score was $>100$ to enhance the specificity of enzyme in $M$. tuberculosis.

The obtained targets genes were further analyzed by UniProt (Universal Protein Resource) (http://www.uniprot .org/) database to find out their functions. This is required to find out the surface membrane proteins which could be probable vaccine targets.

\section{Results and Discussion}

3.1. Identification of Unique Pathways and Potential Drug Targets. Tuberculosis (TB) is a major cause of illness and death worldwide, especially in Asia and Africa. Globally, 9.2 million new cases and 1.7 million deaths from TB occurred in 2006, of which 0.7 million cases and 0.2 million deaths were in HIV-positive people [2]. The existing drugs have several shortcomings, the most important of them being the emergence of drug resistance.

No new anti-Mtb drugs have been developed for well over 20 years. In view of the increasing development of resistance to the current leading anti-Mtb drugs, novel strategies are desperately needed to avert the "global catastrophe" forecast by the WHO (World Health Organization). Therefore, computational approach for drug targets identification, specifically for $M t b$, can produce a list of reliable targets very rapidly. These methods have the advantage of speed and low cost and, even more importantly, provide a systems view of the whole microbe at a time. Since it is generally believed that the genomes of bacteria contain genes both with and without homologues to the human host. Using computational approach for target identification it is very quick to produce a desirable list.

In the present study, 5 unique pathways, C5-branched dibasic acid metabolism, carbon fixation pathways in prokaryotes, methane metabolism, lipopolysaccharide biosynthesis, and peptidoglycan biosynthesis with 60 new nonhomologous targets were identified through in silico comparative metabolic pathway analysis of Homo sapiens and M. tuberculosis H37Rv using KEGG database. Pathways which are not present in the Homo sapiens but present in the Mycobacterium are designated as unique pathways. Design and targeting inhibitors against these nonhomologous sequences could be the better approach for generation of new drugs. Thus total 5 unique metabolic pathways have been taken in $M$. tuberculosis (Table 1).

3.2. Identification of Essential Genes. Essential genes are those indispensable for the survival of an organism, and their functions are, therefore, considered a foundation of life. Total 55 enzymes out of all were found to be essential for $M$. tuberculosis life cycle (Table 2). These targets were found to be potential targets and could be considered for rational drug design. Using metabolic pathway information as the starting point for the identification of potential targets has its advantages as each step in the pathway is validated as the essential function for the survival of the bacterium.

3.3. Identification of Drug Target's Functions Using UniProt. The subcellular localization analysis of all supposed essential and unique enzymes of $M$. tuberculosis were evaluated by UniProt server. As it was suggested that, membrane associated protein could be the better target for developing vaccines. After functional analysis unique enzymes involved in cellular components like cell wall, cytoplasm, extracellular region, plasma membrane, and so forth, their biological processes and their functions have been retrieved (Table 3). 


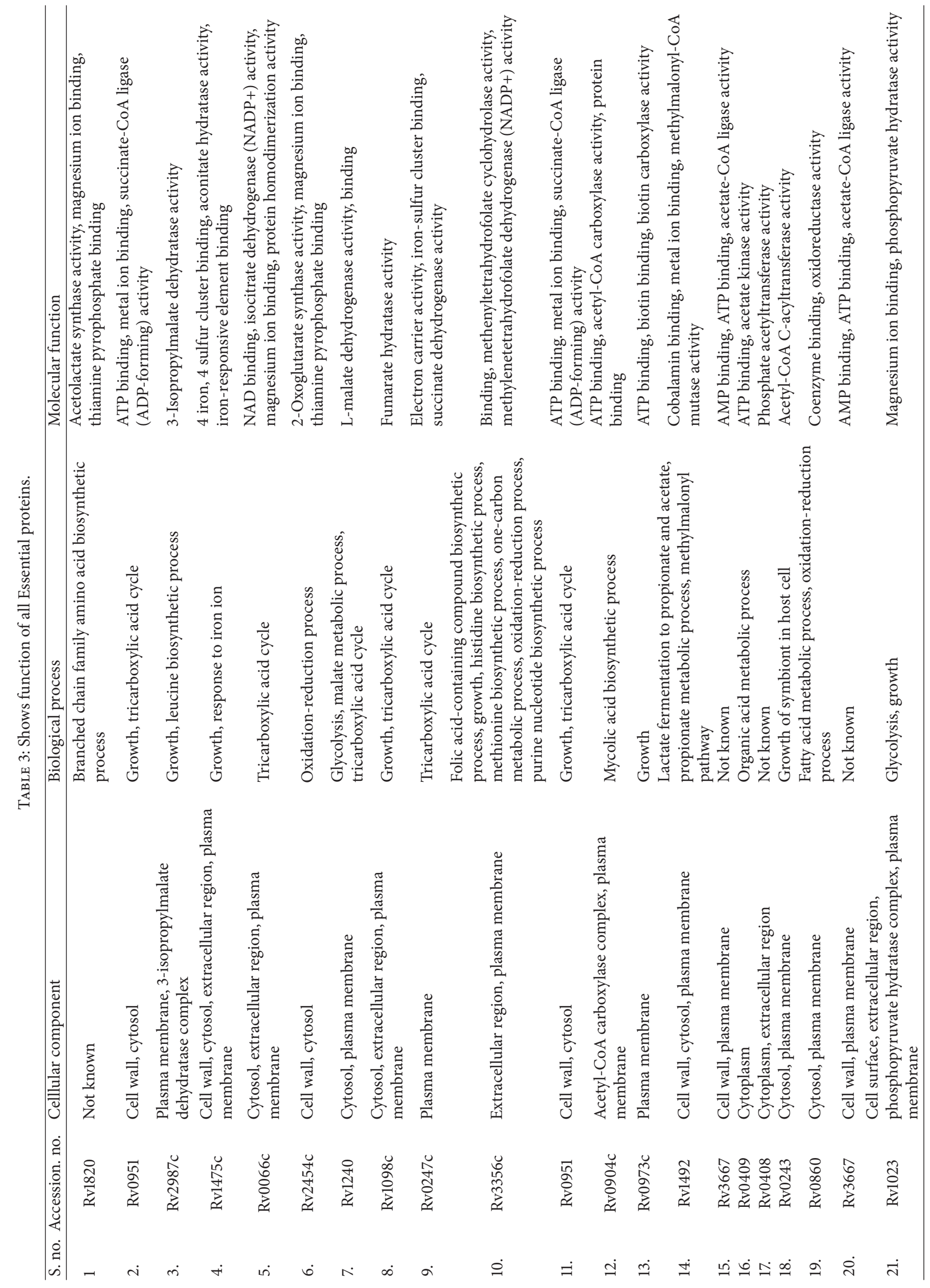




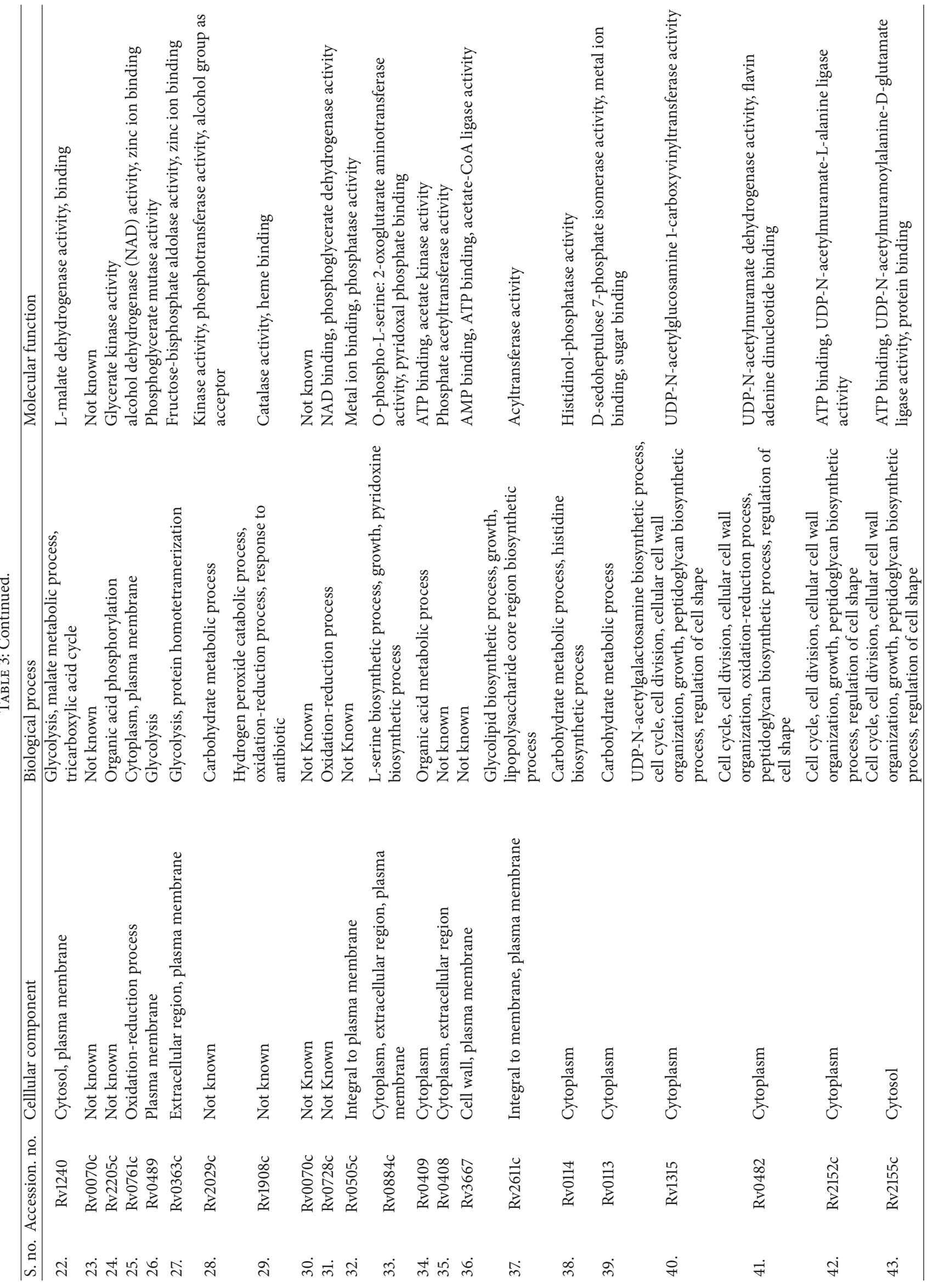




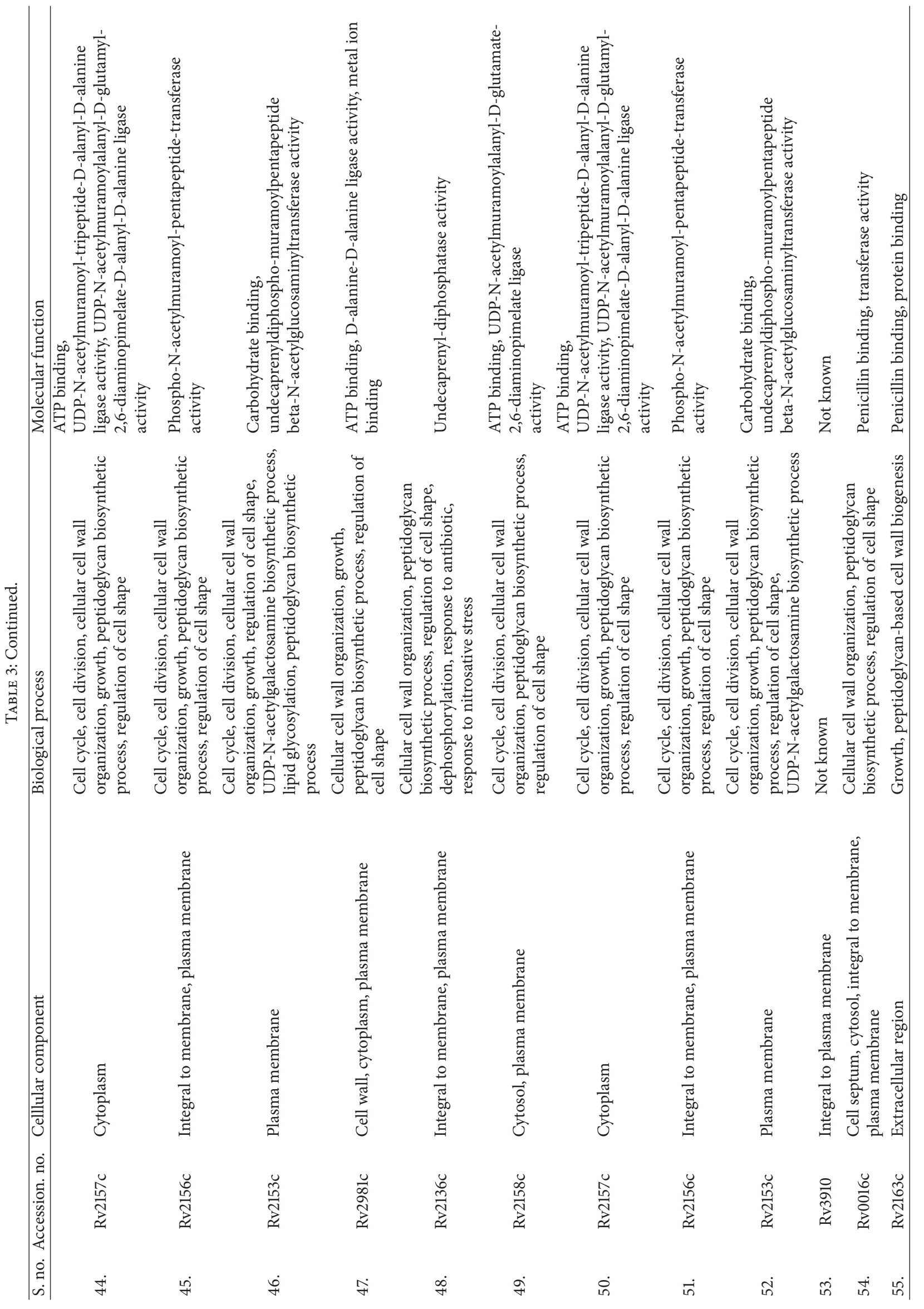


In conclusion, the computational genomic approach has facilitated the search for potential drug targets against $M$. tuberculosis. Use of the DEG database is more efficient than conventional methods for identification of essential genes and it facilitates the exploratory identification of the most relevant drug targets in the pathogen. The current study can be carried forward to design a drug that can block these drug targets. The microorganisms are fast in gaining resistance to the existing drugs, so designing better and effective drugs needs a faster method.

\section{Appendix}

See Tables 1, 2, and 3.

\section{Conflict of Interests}

The authors declare that there is no conflict of interests regarding the publication of this paper.

\section{References}

[1] S. H. E. Kaufmann, "Envisioning future strategies for vaccination against tuberculosis," Nature Reviews Immunology, vol. 6, no. 9, pp. 699-704, 2006.

[2] B. Greenwood, "A global action plan for the prevention and control of pneumonia," Bulletin of the World Health Organization, vol. 86, no. 5, p. 322, 2008.

[3] P. Nunn, B. Williams, K. Floyd, C. Dye, G. Elzinga, and M. Raviglione, "Tuberculosis control in the era of HIV," Nature Reviews Immunology, vol. 5, no. 10, pp. 819-826, 2005.

[4] A. M. Feist and B. Palsson, "The growing scope of applications of genome-scale metabolic reconstructions using Escherichia coli," Nature Biotechnology, vol. 26, no. 6, pp. 659-667, 2008.

[5] A. Dutta, S. K. Singh, P. Ghosh, R. Mukherjee, S. Mitter, and D. Bandyopadhyay, "In silico identification of potential therapeutic targets in the human pathogen Helicobacter pylori," In Silico Biology, vol. 6, no. 1-2, pp. 43-47, 2006.

[6] Ö. Ates, E. T. Oner, and K. Y. Arga, "Genome-scale reconstruction of metabolic network for a halophilic extremophile, Chromohalobacter salexigens DSM 3043," BMC Systems Biology, vol. 5, article 12, 2011.

[7] A. Raghunathan, J. Reed, S. Shin, B. Palsson, and S. Daefler, "Constraint-based analysis of metabolic capacity of Salmonella typhimurium during host-pathogen interaction," BMC Systems Biology, vol. 3, article 38, 2009.

[8] A. M. Feist, C. S. Henry, J. L. Reed et al., "A genome-scale metabolic reconstruction for Escherichia coli K-12 MG1655 that accounts for 1260 ORFs and thermodynamic information," Molecular Systems Biology, vol. 3, article 121, 2007.

[9] N. C. Duarte, M. J. Herrgård, and B. Ø. Palsson, "Reconstruction and validation of Saccharomyces cerevisiae iND750, a fully compartmentalized genome-scale metabolic model," Genome Research, vol. 14, no. 7, pp. 1298-1309, 2004.

[10] A. K. Chavali, J. D. Whittemore, J. A. Eddy, K. T. Williams, and J. A. Papin, "Systems analysis of metabolism in the pathogenic trypanosomatid Leishmania major," Molecular Systems Biology, vol. 4, article 177, 2008.

[11] K. R. Sakharkar, M. K. Sakharkar, and V. T. K. Chow, "A novel genomics approach for the identification of drug targets in pathogens, with special reference to Pseudomonas aeruginosa," In Silico Biology, vol. 4, no. 3, pp. 355-360, 2004.

[12] M. Y. Galperin and E. V. Koonin, "Searching for drug targets in microbial genomes," Current Opinion in Biotechnology, vol. 10, no. 6, pp. 571-578, 1999.

[13] M. Kanehisa, S. Goto, S. Kawashima, and A. Nakaya, “Thed KEGG databases at GenomeNet," Nucleic Acids Research, vol. 30, no. 1, pp. 42-46, 2002.

[14] R. Zhang, H.-Y. Ou, and C.-T. Zhang, "DEG: a database of essential genes," Nucleic Acids Research, vol. 32, pp. D271-D272, 2004 . 

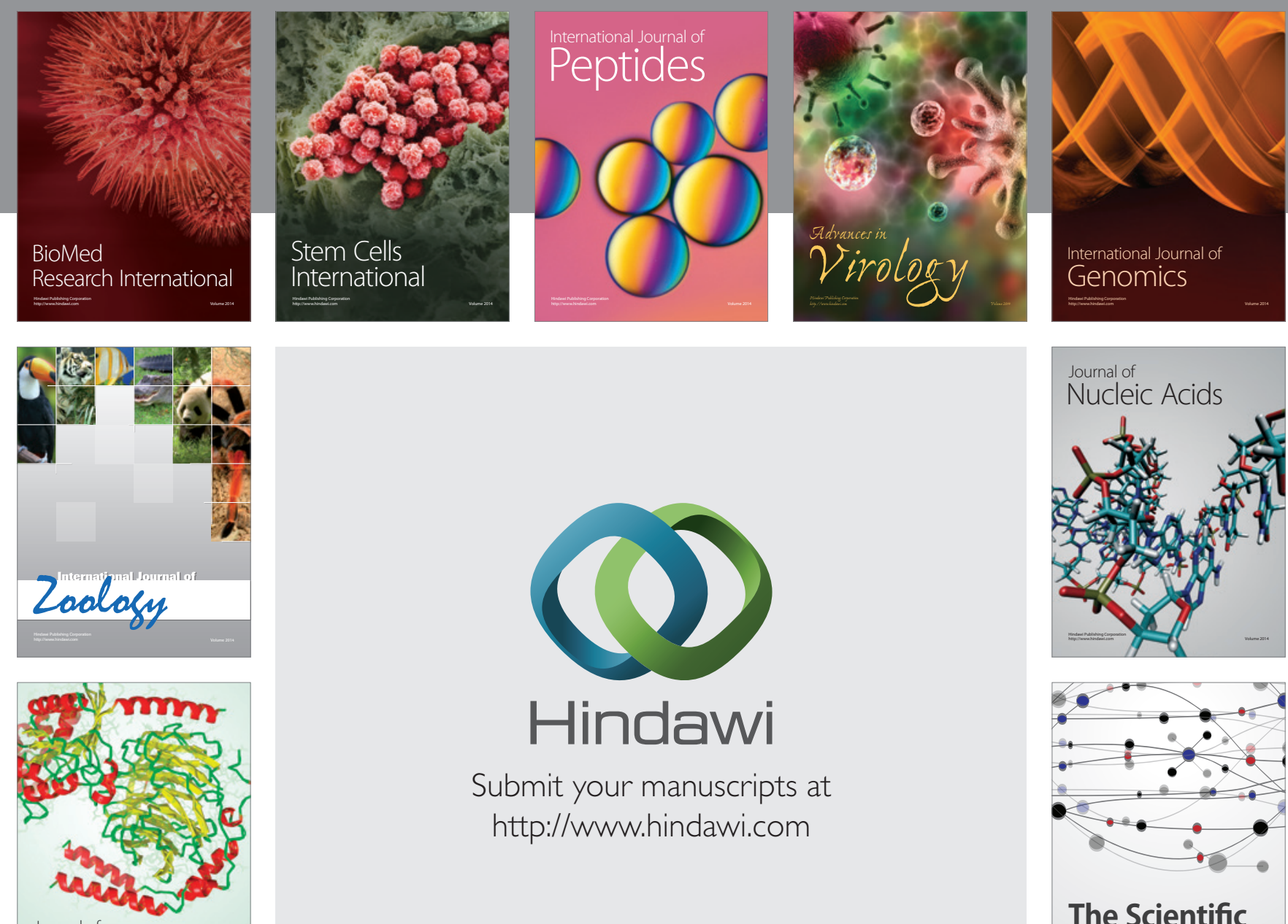

Submit your manuscripts at

http://www.hindawi.com

Journal of
Signal Transduction
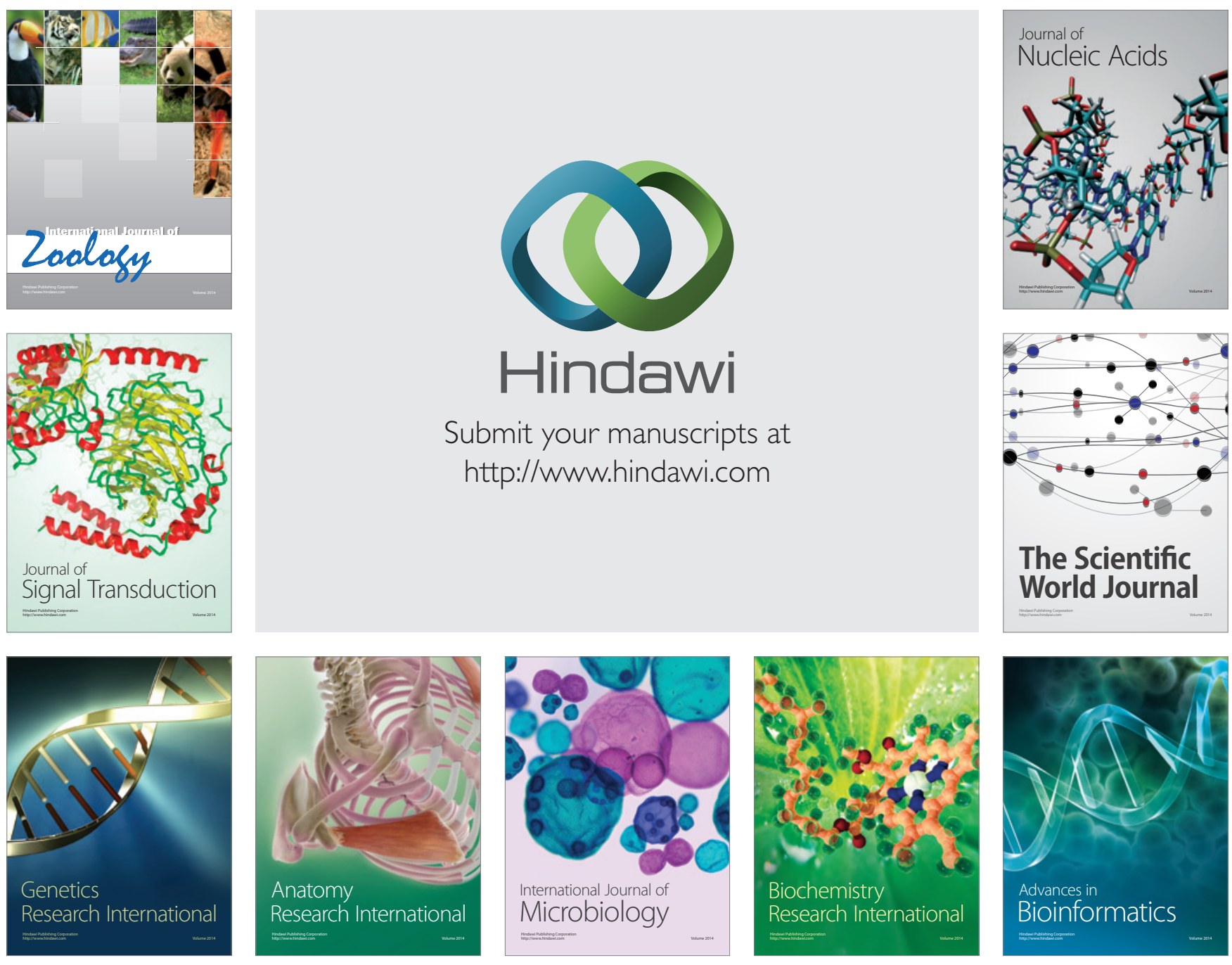

The Scientific World Journal
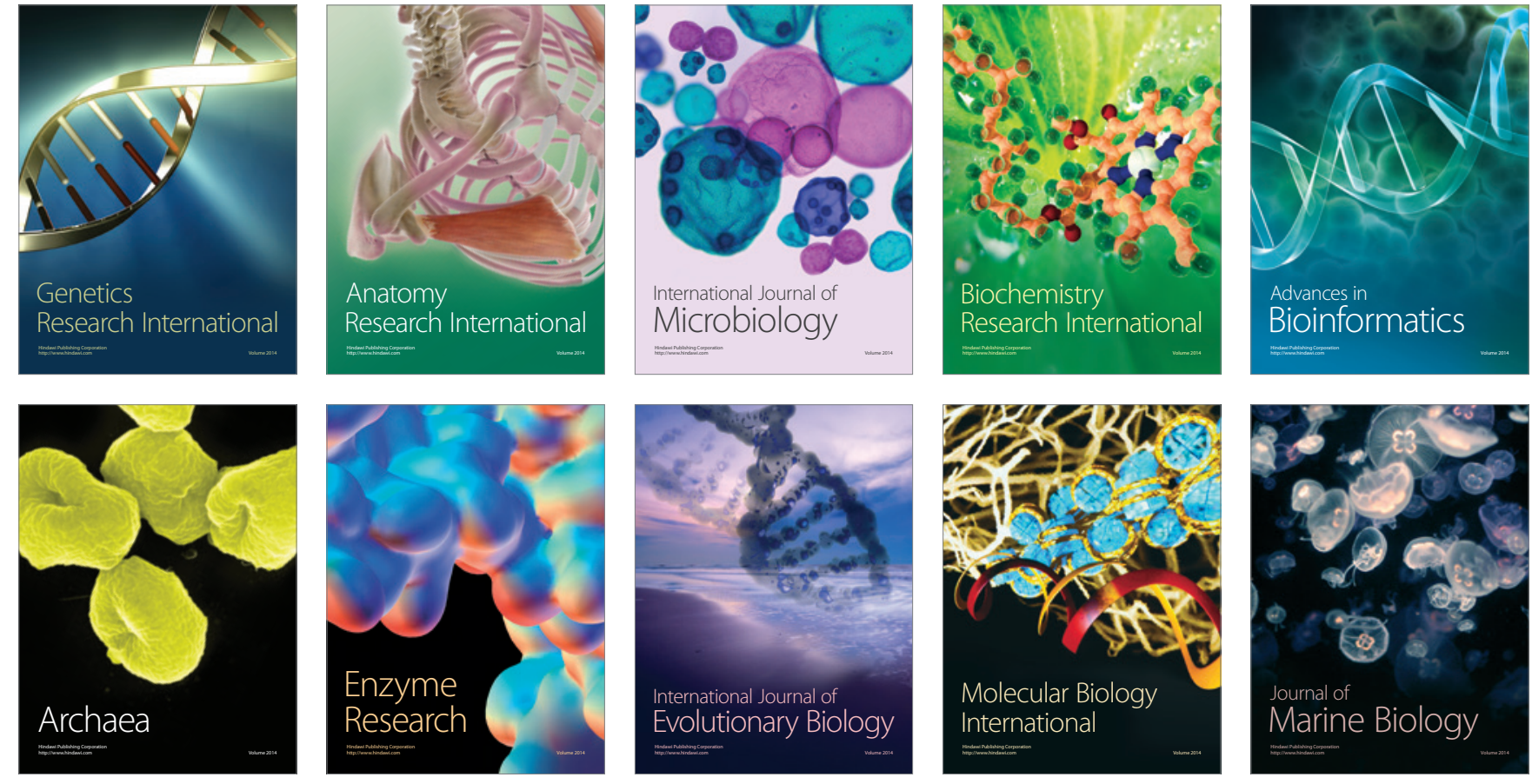